heart and respiratory muscles, as shown by palpitation on movement, sluggish action during repose, and frequent sighing. The nervous system is generally unusually irritable, and the "spirits" are either low or variable.

The pain is related closely to movement. Absent in the morning, it increases in severity as the day wears on, and reaches its acme at night. It is invariably relieved by perfect rest. The spot most commonly affected can ieadily be seen in athletes who are exhibiting feats of strength; it is the sole point in wilich no movement of the skin takes place; viz., where the integument is tied down to bone, and is more or less siretched at every movement of the trunk. The left sido is more commonly affected than the right, because it is weaker. The pain is occasionaily persistent in pregnant women after the fifth month, and seems to be due to stretching of the skin.

I conclude, therefore, that inframammary pain is due to movement disproportioned to strength. We may call it myalgia, neuralgia, or any other algia that we like, without harm, so long as the name involves no wrong system of therapeutics. As the symptom is due to movement, the relief must be sought in repose; and counterirritants, anodynes, ete., are rarely of service, except in so far as they compel quiescence. strapping with a sufficient number of strips to insure immobility of the side is an instant cure. But, as few yersons like to go about thus crippled, a more satisfactory method of treatment is solicited. This is to be sought by endeavouring first to save the patient's strength, and secondly to increase it. In carrying ont t:e détials, each practitioner can adopt the plan he likes best; but of one point he may be assuredviz., that, as a general rule in these casos, air withont cxucise is better than air and exercise. Even in Dr. I'ther's case, the cure was not effected without "bodily repose", though the air and exercise he took increased his health.

\section{CONTRIBUTIONS TO DENTAL SURGERY.}

By Sxmun Adons Parker, Rsq., Licentiate in Dental

Surgery of the Royal College of Surgeons of Eng-

land; Surgeon-Dentist to the Queen's Hospital, and

Senior Surgeon-Dentist to the Birmingham Dental

Dispensary.

Case I. Facial Neuralgia of Twelve Years' Standing, Cured by the Removal of Diseased Teeth. J. Johnstone, aged 43, was admitted to the Dental Dispensary in February 1863, suffering from a violent attack of facial neuralgia, which had existed for a space of twelve years with greater or less severity.

During the last three months previous to my seeing him, the pain, which had been of an acute character, had come on regularly in the evening, and had increased in severity till nearly midnight; when it had gradually subsided into a dull heavy pain, continuing unizterruptedly till the following evening, when the same thing was repeated; and this without variation night after night. The patient also complained of a burning pain on the top of the head, and of a little intermittent pain in the ears. Previously to the occurrence of the neuralgic pains with such severity, the patient had suffered much from his teeth; but, as in numerous cases which I have already published, the pains in the teeth had sub. sided before those of a neuralgic character supervened. The patient attributed his sufferings not to his teeth, but to tic douloureux.

I examined his mouth, and found the teeth for the most part sound, of a slight yellow colour, indicative of strength, and non-liability to decay like those of a pearly white character. However, the stumps of the first and second superior left bicuspid and the second inferior right molar were remaining in the jaw; and the inferior right dens sapientiæ was much decayjed. On the sixth day of February 1863, I removed all the stumps above mentioned. On the $10 \mathrm{th}$, the patient was somewhat better, but still experienced much pain. I then removed the dens sapientiæ.

The patient saw me on the 18th, and informed me that he had been perfectly free from pain since the last operation; and I know he has continued so up to the present time.

Case Ir. Facial Nevralgia Cured by the Removal of Diseased Stumps. Noveruber 6th, 1863. J. Wood, aged 49, had been suffering a long time from neuralgic pains in the head, which had visited him in such violent paroxysms that he became for a time perfectly giddy, and his vision was somewhat impaired thereby.

He had been in this state three years, during which time he had been under medical treatment, and had taken a variety of medicine without much benefit, and had been ultimately consoled by the information that the pain would continue for the remainder of his life. This patient always had an idea that the pains started from the lower jaw, where two or three old stumps were; but the mouth had never been examined.

From the condition and appearance of the stumps, I was induced to believe that much benefit, if not an ultimate cure, might be efrected by their removal; to which the pationt readily consented. I removed four stumps, which were all that remained in the lower jaw ; the other teeth being in a tolerably good condition.

I saw him three times after the removal of the stumps, and found that the pain had gradually subsided, and had finally left him.

CASE III. Morbid Growth of the Gums Caused by Discased Stimps : Removal of the Stumps, and Ultimate Cure. Mr. B., aged 30, consulted me in July 1863; respecting his gums and palate, which had been in an intlamed and otherwise diseased condition for four months.

I found upon examination, that the gums were very thick and spongy; that they bled profusely upon the slightest touch, and discharged a thin matter from their maroins, which rendered his breath very offensive. This condition of things had extended to the palate and timoat, and had rendered them very mach inflamed. In the centre of the palate was an abscess, which, when opened, discharged a quantity of thick pus. The patient had lost the crowns of nearly every tooth ; the stumps were for the most part loose, and on tilat account had been a cause of great irritation, and had rendered the patient's condition daily worse.

I removed four or five (as many as he was able to bear at one time) stumps and freely scarified the gums, more particularly between the teeth; and ordered a wash for the mouth composed of

Tinct. myrrhæ $j^{\mathrm{iv}}$; infusi rosæ comp. $\zeta$ viii ; acidi nitrici diluti $m x x$.

To be used three times a day.

This wash I invariably order in cases where the gums are at all inflamed and spongy, and always have found that patients have derived great benefit from it. I also ordered him to take a small quantity of lime-juice internally every day, and to be careful as to his diet.

I saw the patient a few days after the operation, and found tha $\lrcorner$ a more rapid improvement had taken place than $I$ had anticipated. The palate and throat were free from all inflanmation, and the abscess was fast closing up. The gums, although not by any means well, had an improved condition about them 
which gave promise of an early cure. On the occasion of this visit, I removed the remainder of the stumps, and ordered him to continue the wash and lime-juice.

At the expiration of a fortnight, I again saw the patient, and during the intervening time, he had been getting gradually better, and the mouth, in which, however, very few teeth remained, was cured.

CASE IV. Strabismus Caused by a Decayed and Aching Temporary Lower Molar: Removal of the Tooth: Instantaneous Cure of the Disease. This may, at first sight, seem a strange case. It is the first of its kind that has ever come under my notice, and was remarkable in two respects ; first, for its sudden appearance and secondly, for its sudden disappearance. The case may be related in a very few words.

A child, four and a half years old, came under my care at the Queen's Hospital. She was brought by her mother, who stated that, seven days previous to my seeing her, she was attacked with a violent pain in the first inferior temporary molar, and at the same time the left eye completely turned and became fixed. The tooth was not aching at the time I saw her, but it was very much decayed, and I extracted it. In twenty-four hours after the operation the eye began to turn, and in three days it was perfectly straight.

In this case, there could be no question as to the cause of the strabismus, and none as to the remedy and cure.

CAse v. Lesion Produced by a Coming Wisdom Tooth. Georgina Maynard, aged 29, was admitted an out-patient at the Queen's Hospital, October 7th, 1863, under the care of Mr. Gamgee, sufiering from a large, hard, and extremely painful swelling on the whole of the right side of the face, more particularly over the upper molar teeth. She stated she first felt pain six months previously, when she noticed a swelling. She did not do anything for it till some seven weeks previously to coming to the hospital, when, on account of the great pain she experienced, she fomented the parts daily with poppy heads. Mr. Gamgee ordered a linseed meal poultice to the swelling, and a mixture of acid and bark to be taken internally.

October 14th. At this time, in consequence of the size, stifiness, and extreme tenderness of the swelling, she was unable to open her mouth. The medicine was continued, and an embrocation of two drachms of tincture of hyoscyamus and six drachms of oil of almonds ordered to be applied over the swelling.

October 17th. The poultice was repeated, and she was ordered to see the surgeon-dentist.

October 29th. This was the first time I saw the patient; and, finding that she could open the mouth but very little, I ordered a fomentation of poppy-heads to be used as hot as she could bear it inside the mouth.

November 1st. An abscess had burst in the mouth since using the fomentation, and she could open her mouth very much better. The poppy-head fomentation and mixture were continued.

November 9th. The swelling had been considerably diminished. She could open her mouth almost without pain, though she still suffered much pain at the back of the jaw. I now carefully examined the mouth, and found that the whole of the mischief was caused by a coming wisdom tooth. The gum over the tooth I freely lanced by making a crucial incision.

November 16th. The wisdom tooth had made great progress ; the swelling was entirely gone down, and the pain gradually subsiding. The tonic mixture was continued.

November 23rd. She was discharged cured. The wisdom tooth was nearly through the gum.

REMARKs. This is the fourth time I have contributed cases of dental interest to the medical journals; and in doing so, I have selected those cases from my note-book, which are of importance, rather, perhaps, to the medical than to the dental profession, on account of their coming under the notice and treatment of the former class, before they are seen by the latter.

I have endeavoured from time to time, to point out and impress upon my readers, the great importance of the mouth being at all times carefully examined by the medical practitioner, whenever cases may come under their observations, of facial neuralgia, and tic doulourenx, for it very rarely occurs that the teeth have not played some prominent part in bringing on the mischief.

For instance, in case No. 1 , the disease had extended over twelve years, without any examination having ever been made of the teeth; and in case No. 2 , it was the patient's own opinion, that prompted him to seek advice, after he had been informed that he should never be well arain.

In most of the cases I have published, it will be seen that the pains alluded to are confined to one $\mathrm{Oi}^{*}$ two particular spots, either in the temples, over the orbit, in the ears, or along the lower jaw; sometimes the pain extends down the neck to the shoulders.

It is sometimes very difficult, without a considerable amount of experience, for the dentist to distinguish, or rather to diagnose, the particular tooth or teeth that may be the primary canse of the mischief; for, as I have stated before, when the pain becomes seated in any of the parts above mentioned, little or no toothache proper is experienced; hence the unwillingness of the patient to have any operations performed upon the teeth.

The pain arising from toothache is nearly always centered in and around the diseased organ; while neuralgic pains, arising from the same cause-viz., diseased teeth or stumps-are situated at some distant part.

We find occasionally, not frequently, upon examining the mouth carefully for some exciting cause, that every tooth appears perfectly sound, though still some diseased condition may be lurking in one or more of them, which may be generally discovered, by gently tapping each tooth with an instrument, or, as Professor 'Tomes suggests, by throwing a jet of cold water from a syringe upon them.

When the pain appears to arise from a sound tooth (there being no diseased ones in the jaw), every means should be adopted by the medical practitioner previous to extraction-aperients, tonies, and local depletion being especially valuable.

As regards the injury done to the general system by a diseased condition of the mouth, a few remarks will not be out of place. Proofs without number have been given, that a diseased or abnormal condition of the teeth may very seriously, and indeed sometimes fatally, affect the general health of the sufferer therefrom ; and it must be no less evident to unprofessional than to professional observers, that ill health is the frequent cause of bad teeth. One would have thought, as in this case, a reciprocal action is so plainly evident, that it would naturally be made the basis for the exertions of both medical and dental efforts, to restore the general health to its normal natural tone.

There is, however, another consideration which may serve to put the danger of diseased teeth affecting the general health in a yet stronger light. Their important connection with the organs of digestion, by whose abnormal condition the whole body becomes weak and disordered, is surely enough to suggest that by attention to those teeth the very fountainhead of the malady may be reached, and its further progress arrested.

It is well known that even sound teeth in every stage of their development have been the cause of 
serious and at times of fatal mischief. They have been the cause of infantile convulsions and consequent death; and surely in a diseased condition they may naturally be expected to interfere with the health of the adult, who is arrived at that period of life at which he is more at the mercy of their mischievous tendencies, though in his case the evil may proceed in a subtler form, and with a slower pace.

\section{SOME ACCOUN'T OF THE OPERATIONS} PRACTISED IN THE NINETEENTH

CEN'TURY FOR 'THE RELIEF OF TENSION OF THE EYEBALL, GLACCOMA, ETc.

By James Vose Solomon, F.R.C.S., Surgeon to the Birmingham and Midland Eye Hospital. [Concluded from page 92 of last volume.]

Diseased States in which the Operation has proved Useful. On March 1st, 1860, I operated upon a much disorganised and tense eye; and on the 31st, upon a case of near-sightedness (myopia), complicated by choroido-retinitis. The pupillary margin of the iris "was drawn slightly towards the lens, the other part of the membrane being arched forwards, except at its ciliary origin."

The effect upon the accommodation of the eye and congestive symptoms proved so remarkable, that I was encouraged to submit other myopic patients to a similar plan, and obtained markedly beneficial results.

The theory of treatment in this class of cases was based upon the opinion, at the time generally prevalent in England, and taught in the best text-books of physiology; namely, that the ciliary muscle consisted of a single set of fibres, which in direction were radial, and that the adjustment of the eye to near objects was effected by contraction of the muscle drawing the lens towards the cornea.

I argued thus: if I cut some of these fibres across, the muscle of the lens will be weakened, and the far point for reading will undergo an increase.

As my views upon this subject, with clinical illustrations, have been already given in the Medical Times and Gazette (vol. 1861-62), and will shortly appear in a separate form, it is unnecessary here to do more than notice that, in cases which I have had opportunity of following since the year 1860, the increase of accommodation for large and distant objects, as human features and landscape, has been most completely maintained*; while a very slight contraction has taken place in the reading distance.

In other words, the myopia, as regards distance, has been permanently improved and arrested; a slight progress only having occurred in respect to near ob. jects.

Another point of interest was presented in two cases of extreme myopia, who, having been much improved by the operation, resumed, after a time, the use of their deep concave spectacles for looking at near and far objects. In each instance, the nearsightedness returned to what it was before surgical treatment.

Do not these facts obtain interest from their relation to the mechanism which accommodates the eye to different distances? Are they not suggestive of the existence of some active agency by which the organ is adjusted for objects placed beyond twenty

* Many writers consider the eye to be passive when viewing landecape, and that accommoriation or adjustment only comes into play when near objects are viewed. 'T'hey thercfore object to speak of accommodation for distance.

+ According to Douders, the natural tendency of myopia is to advance. feet? The time, we would fain believe, is not remote, when a solution of some of the several problems connected with the subject of optical accommodation and refraction will be attempted by a recourse to intraocular myotomy in suitable cases.

A word of caution on the selection of cases. Instances of hypermetropia and astigmatism, diseases which are sometimes relieved by concave glasses, and which might, on cursory examination, be confounded with near-sightedness, must be carefully excluded from operation.

To proceed with the narrative. In the same year and month, I operated upon a case of acute choroiditis, complicated by great tension and myosis. The first two conditions were reduced by the treatment, and vision restored; and I would here repeat the opinion expressed at the meeting of the British Medical Association in London (1862); namely, that I am unacquainted with any "surgical measure, equally safe and easy of execution, which exerts the same amount of curative power in cases of subacute and chronic choroiditis," as intraocular myotamy.

In April 1860, I treated instances of glaucoma, with very satisfactory results. In the glaucomoid tension well known to practical ophthalmic surgeons, as occasionally following the operation of cataract extraction, where vitreous, even though small in quantity, has been lost, and the iris obliterated from view at the centre of the cicatrix, the operation under consideration removes the tension and restores clear vision. In one case, the tension was not completely overcome until after the incision was repeated, and slightly extended in length.

Previous to adopting this method, I made trial of division of the ciliary structures at a right angle with the cornea, selecting the point where they were continuous with the coloboma. In none, were the symptoms ameliorated; in two, so mach aggravated as to suggest the expediency of an immediate enucleation of the globe.

I recommend that the intraocular myotomy should always include the base or pillars of the widest part of the iris. Mr. Teale (Britrish Medical Jouranal, April 9th, 1864, page 404) appears to have practised, at the suggestion of $\mathrm{Mr}$. Bowman, a somew hat similar, if not an identical, plan of treatment, in two cases in which exalted tension was consequent upon a needle operation for cataract. The first of Mr. Teale's cases occurred nearly a year after my papers had appeared in the Medical Times and Gazette.

In the glaucomoid state, which sometimes forms a sequela of violent injury to the eyeball, when complicated by dislocation of the lens deeply into the vitreous, no surgical treatment can be relied on as curative of the tension and prophylactic of the occurrence of sympathetic ophthalmia.

Discouraged by the results which $I$ had witnessed in my own practice and that of others, I witheld all surgical interference in the last.case that came under my care. The irritation subsided, and no serious sympathetic mischief followed in the fellow organ. The patient was nearly 60 years of age.

In May 1860, the operation-by removing tension from an eye in which the pupil was closed and the iris bossulated (synechia annularis) - cured a sympathetic irritation of the fellow organ, that had existed a year, and which, at the time of treatment, rendered the reading of small type impossible. (Vide Medical Times and Gatette, vol. 1661, p. 327.)

The case derived additional interest from showing that the irritation due to an exalted intraocular tension may be imparted to a sound eye.*

* This fact is, I believe, now (September 1864) conceded, quoad glaucoma, by the iridectomy school. 